\title{
The Correlation of Quantum Mechanics and Whitehead's Philosophy
}

It is a remarkable characteristic of the history of thought that branches of mathematics, developed under the pure imaginative impulse, thus controlled, finally receive their important application. Time may be wanted. Conic sections had to wait for 1800 years. In more recent years, the theory of probability, the theory of tensors, the theory of matrices are cases in point.

Alfred North Whitehead, Process and Reality

ThE ONTOLOGiCAL INTERPRETATION of quantum mechanics explored thus far in this essay can be distinguished from other interpretations by two primary characteristics: First, it is an interpretation that attempts to describe, via imaginative hypothetical deduction, the form among the facts of experience, rather than both the form and the facts, as is the case with many other interpretations of quantum mechanics-those, for example, that attempt to account for the actualization of potentia by way of the mechanics. The existence of facts by this interpretation is accepted a priori, such that the mechanics and its interpretation both presuppose and anticipate the facts of actuality as described in the quantum mechanical evolution of system states. The "problem of the actualization of potentia" is thus no problem at all by this interpretation, which merely seeks to describe the underlying form, and implications, of the quantum mechanical process by which actuality evolves to actuality, mediated by given potentia.

Second, it is an interpretation that, via the decoherence effect and its required mutual interrelations between the measured system and all facts environmental to it, explicitly makes use of the requirements 
of logic, coherence, and universal applicability and adequacy typically lacking in most interpretations of quantum mechanics. For these desiderata are satisfied with the explicit recognition of the universe in its entirety as the only truly closed system to which quantum mechanics might apply, and it is this recognition that guarantees the necessary, mutual interrelations among all facts. The implications of quantum mechanics by this interpretation are thus universal and therefore ontologically significant; and indeed, this interpretation of quantum mechanics constitutes the exemplification of a clear ontological principle, rather than merely an epistemological principle such as Bohr's principle of complementarity. The ontological principle is: Every fact is a determinant in the becoming of every new fact, such that the evolution of any fact entails both temporally prior facts and logically prior potentia as data, and an integration of these data that is unique to that evolution.

The interpretation of quantum mechanics described in Part I is a fundamental physical exemplification of this ontological principle; and given that, one might infer that it is an exemplification of some much broader metaphysical scheme that must flow from this principle. It would have to be a scheme wherein the universe is characterized as an ongoing process of actualizations (described by quantum mechanics as an historical route of state evolutions of $|\Psi\rangle$ ). Each actualization is itself a process, comprising the following phases as exemplified by quantum mechanics:

(i) An initial phase, consisting of the integration of all facts relative to a particular fact belonging to a particular subsystem of facts (e.g., the "indexical eventuality" belonging to the measuring apparatus) into potential forms or states. Since the process of actualization is described mechanically as an "evolution" of the state of the system of facts relative to a particular fact, the latter must therefore have two natures: (a) that of the subject of the state evolution, partially characterized by its inclusion in and relation to $S_{\text {init }}$ (e.g., in quantum mechanics, the system state always evolves relative to the indexical eventuality and its associated preferred basis); (b) that of the product of the state evolution, partially characterized by its inclusion in and relation to $S_{\text {fnal }}-\mathrm{a}$ novel integration of facts which includes the newly evolved indexical eventuality. The relativity of this integration is both objective and subjective. It is objective in that it is an integration of facts, and it is subjective in that the uniqueness of the indexical 
eventuality, together with the objective actuality of the facts interrelated, conditions the particular form of the integration. The latter is reflected in quantum mechanics by the fact that a preferred basis is always associated with any indexical eventuality.

(ii) A supplementary phase, whereby potential facts incapable of integration are eliminated via negative selection, yielding a reduced matrix of mutually exclusive, valuated potential integrationsthat is, a matrix of potential states or potential forms of facts, valuated as probabilities. These forms are subjective insofar as they are integrations of facts relative to a unique indexical eventuality; but they are also objective insofar as they are integrations of facts.

(iii) The actualization of one of these integrations according to the valuations qualifying each, in satisfaction of the evolution and its aim-the latter as evinced by the probabilistic nature of these valuations.

Readers familiar with the cosmological scheme developed by Alfred North Whitehead in his "philosophy of organism" have likely already inferred a number of correlations between the Whiteheadian scheme and the interpretation of quantum mechanics described in Part I. The explication of these correlations is the task of the remainder of this book.

It should be noted that the development from 1924 to 1930 of the "new" quantum theory of Heisenberg, Bohr, Schrödinger, et al., and its philosophically troublesome innovations-many of them hashed out at the Solvay Conferences of 1927 and 1930 — took place during the same years that Whitehead developed his cosmological scheme, presented in its most complete, systematic form-Process and Reality-in his 1927-1928 Gifford Lectures, published in 1929. (Whitehead had presented an earlier version of this scheme in the 1925 Lowell Lectures, as well as in Science and the Modern World, published the same year.) One is left to wonder, then, whether Whitehead was aware of the troubling philosophical implications of the "new" quantum mechanics-as opposed to the "old" quantum mechanics consisting of Einstein's and Planck's theories of quantized transference of electromagnetic energy combined with Bohr's 1913 model of the atom.

Whitehead occasionally refers to the quantum theory in presenting his metaphysical scheme, and it is clear that some, if not most, of these references refer to the "old" quantum theory: 
The treatment of cosmology in the philosophy of organism . . contains the discussion of the ultimate elements from which a more complete philosophical discussion of the physical world - that is to say, of nature-must be derived. In the first place an endeavour has been made to do justice alike to the aspect of the world emphasized by Descartes and to the atomism of the modern quantum theory. Descartes saw the natural world as an extensive spatial plenum, enduring through time. Modern physicists see energy transferred in discrete quanta. ${ }^{1}$

But Whitehead also refers to concepts inherent in the quantum theory as developed by Heisenberg, Bohr, Schrödinger, et al.- the "new" quantum theory, which is formulated, for example, in precisely the same terms Whitehead uses in the quotation from Process and Reality that begins this chapter: Namely, "the theory of probability, the theory of tensors, the theory of matrices." (Recall that the combined Hilbert spaces representing a composite system-apparatus-environment are tensor product spaces; and that sets of probability-valuated potentia are grouped into matrices, etc.) His references to this terminology aside, Whitehead also refers to the "new" quantum theory in terms of two fundamental conceptual innovations primarily associated with it: First, the refutation of fundamental materialism as given in Whitehead's "fallacy of misplaced concreteness":

This fallacy consists in neglecting the degree of abstraction involved when an actual entity is considered merely so far as it exemplifies certain categories of thought. ${ }^{2}$

Material substance is one such category, through which the fallacy of misplaced concreteness (and the related "fallacy of undifferentiated endurance") has led to the doctrine of materialism in which

the notion of continuous stuff with permanent attributes, enduring without differentiation and retaining its self-identity though any stretch of time however small or large, has been fundamental. The stuff undergoes change in respect to accidental qualities and relations; but it is numerically self-identical in its character of one actual entity throughout its accidental adventures. The admission of this fundamental metaphysical concept has wrecked the various systems of pluralistic realism. This metaphysical concept has formed the basis of scientific materialism. ... But this materialistic concept has proved to be as mistaken for the atom as it was for the stone. ${ }^{3}$ 
Whitehead here refers to the quantum mechanical description of the atom-a description which many physicists, in their debates concerning the proper formulation of the "new" quantum theory, attempted to fit into a materialistic framework. Of these attempts Heisenberg, echoing Whitehead's words above, writes: "It would, in their view, be desirable to return to the reality concept of classical physics or, to use a more general philosophic term, to the ontology of materialism. They would prefer to come back to the idea of an objective real world whose smallest parts exist objectively in the same sense as stones or trees exist." According to the Copenhagen Interpretation, Heisenberg continues, "modern atomic theory no longer allows any reinterpretation or elaboration to make it fit into the naive materialistic conception of the universe."4 Thus, as Whitehead points out, "the field is now open for the introduction of some new doctrine ... which may take the place of the materialism with which, since the seventeenth century, science has saddled philosophy." 5

What has vanished from the field of ultimate scientific conceptions is the notion of vacuous material existence with passive endurance, with primary individual attributes, and with accidental adventures. Some features of the physical world can be expressed in that way. But the concept is useless as an ultimate notion in science, and in cosmology. ${ }^{6}$

The simple notion of an enduring substance sustaining persistent qualities, either essentially or accidentally, expresses an abstraction useful for many purposes of life. But whenever we try to use it as a fundamental statement of the nature of things, it proves itself mistaken. ${ }^{7}$

The second conceptual innovation of the "new" quantum theory to which Whitehead refers is closely related to the first: the concept of concrescent state evolution, wherein the final state of a system of facts evolves from the interrelations of its potential facts with the antecedent facts described by the initial system state. This is an exemplification of Whitehead's Ontological Principle:

Every condition to which the process of becoming conforms in any particular instance, has its reason either in the character of some actual entity in the actual world of that concrescence, or in the character of the subject which is in process of concrescence. ${ }^{8}$

The actual world is the "objective content" of each new creation. ${ }^{9}$ 
Whitehead thus characterizes these interrelations between concrescing potentia and the world of actualities antecedent to them as having, in his words, a "vector character" 10 in the sense that each potential fact in the process of concrescence "has its reason" in some particular antecedent fact or facts. ${ }^{11}$ Further, the serial evolution of these concrescences-the historical route of state evolutionsmanifests itself in the "ultimate vibratory characters of organisms and to the potential element in nature": 12

The atom is only explicable as a society with activities involving rhythms with their definite periods. Again the concept shifted its application: protons and electrons were conceived as materialistic electric charges whose activities could be construed as locomotive adventures. . . . The quanta of energy are associated by a simple law with the periodic rhythms which we detect in the molecules. Thus the quanta are, themselves, in their own nature, somehow vibratory; but they emanate from the protons and electrons. Thus there is every reason to believe that rhythmic periods cannot be dissociated from the protonic and electronic entities. ${ }^{13}$

Similarly, in Modes of Thought Whitehead writes, "There is a rhythm of process whereby creation produces natural pulsation, each pulsation forming a natural unit of historic fact."14 The natural unit of historic fact, as applied to quantum mechanics, is the newly evolved, fully determinate system state-a "society" of facts actualized from among a matrix of potential states that have themselves evolved from a society of antecedent facts; and the rhythm is the alternation between the newly evolved, unitary, actual system state and the multiplicity of antecedent facts (and their associated potentia) from which the novel state evolves. Thus, the "many" antecedent facts and their associated potentia become "one" novel state (a novel fact), and are increased, historically, by one-a process which repeats itself "to the crack of doom in the creative advance from creature to creature." 15

Whitehead's references to the quantum theory as an exemplification of his cosmological scheme, then, pertain to three related concepts-the first one best associated with the "old" quantum theory of Planck and Einstein as applied to Bohr's 1913 atomic model, and the other two best associated with the "new" quantum theory of Heisenberg, Schrödinger, Bohr, et al., typically referred to as the Copenhagen Interpretation: 
1. ("old" quantum theory): The transference of electromagnetic energy in discrete quanta and the "vector" relationship between such transference and photonic emissions (Einstein's photoelectric effect). "The mysterious quanta of energy have made their appearance, derived, as it would seem, from the recesses of protons or of electrons. Still worse for the concept, these quanta seem to dissolve into the vibrations of light. Also the material of the stars seems to be wasting itself in the production of the vibrations." 16

2. ""new" quantum theory): The fallacy of misplaced concreteness (and undifferentiated endurance) as it applies to the doctrine of materialism-successful since the seventeenth century, but unable to accommodate the quantum theory. ${ }^{17}$ The latter instead characterizes material substance in terms of "systems" of rhythmically evolving actualities, such that "The atom is only explicable as a society with activities involving rhythms and their definite periods." Thus, "we diverge from Descartes by holding that what he has described as primary attributes of physical bodies, are really the forms of internal relationships between actual occasions, and within actual occasions. Such a change of thought is the shift from materialism to organism, as the basic idea of physical science." 18 This concept is closely related to the next.

3. ("new" quantum theory): The "vector relationship" between potential facts (or systems/societies of facts) in the process of actualization and all antecedent facts, such that the latter contribute in a specific way to the definiteness of the former, in exemplification of the ontological principle: Every actualization of a potential fact is partially determined by its specific relations with all facts antecedent to it, constituting the entire extant universe relative to the actualization at hand. In this way, "the actual world is the 'objective content' of each new creation." 19 The universe is thus characterized as a closed system, as required by any ontologically significant interpretation of the Schrödinger equation, such that any actualization within it necessarily involves all other actualities. This is reflected in Whitehead's "Principle of Relativity," according to which every actuality is a potential determinant in the becoming of every new actuality-a principle closely related to his Ontological Principle. The latter is echoed by Heisenberg when he writes, "the transition from the "possible' to the 'actual' takes place as soon as the interaction of the object with the measuring device, and thereby with the rest of the 
world, has come into play" (emphasis added). ${ }^{20}$ Similarly, the epistemological implications of this fundamental characterization of nature given in the "new" quantum mechanics — the Heisenberg uncertainty relations and Bohr's principle of complementarity-are echoed by Whitehead when, in his commentary on the quantum theory cited above, he writes: "We are now approaching the limits of any reasonable certainty in our scientific knowledge."21

Whitehead's references to the quantum theory in Process and Reality always reflect what appears to be a distinction between the implications of the "old" quantum theory and those of the "new" quantum theory. Consider the following passages, where concepts best pertaining to the "old" quantum theory are printed in italics, and those best pertaining to the "new" quantum theory are printed in boldface:

In the language of physical science, the change from materialism to "organic realism"- as the new outlook may be termed-is the displacement of the notion of static stuff by the notion of fluent energy. Such energy has its structure of action and flow, and is inconceivable apart from such structure. It is also conditioned by "quantum" requirements. These are the reflections into physical science of the individual prehensions, and of the individual actual entities to which these prehensions belong. Mathematical physics translates the saying of Heraclitus, "All things flow," into its own language. It then becomes, All things are vectors. Mathematical physics also accepts the atomistic doctrine of Democritus. It translates it into the phrase, All flow of energy obeys "quantum" conditions.22

And:

[If we] remember that in physics "vector" means definite transmission from elsewhere, we see that this metaphysical description of the simplest elements in the constitution of actual entities agrees absolutely with the general principles according to which the notions of modern physics are framed. The "datum" in metaphysics is the basis of the vector-theory in physics; the quantitative satisfaction in metaphysics is the basis of the scalar localization of energy in physics. ${ }^{23}$

And:

In the language of science, [the philosophy of organism] describes how the quantitative intensity of localized energy bears in itself the vector marks of its origin, and the specialities of its specific forms; it 
also gives a reason for the atomic quanta to be discerned in the building up of a quantity of energy. In this way, the philosophy of organism-as it should—appeals to the facts. ${ }^{24}$

It should be emphasized here that the "new" quantum theory is, in one sense, a more complete systematization of the "old" quantum theory such that the old theory is wholly subsumed within the new theory; therefore, the preceding passages, though they might best apply to either the "old" or the "new" quantum theory, fundamentally apply to both. In the last passage, for example, the "vector marks of [a localized energy's] origin, and the specialities of its specific forms" could also apply to the photoelectric effect of the "old" quantum theory, where energy in the specific form of an emitted photon has a vector relationship to the electron-nucleus interrelation originating the emission.

Discussions of the applicability of Whitehead's philosophy to quantum mechanics typically address whether or not the "new" quantum theory and the "Copenhagen Interpretation" of this theory, formulated contemporaneously with Whitehead's development of his cosmological scheme, might have perhaps influenced the latter in some way. Abner Shimony, for example, states that "Whitehead never refers to the new quantum theory, and it would be unreasonable to expect that even so imaginative a philosopher and scientist as he could have anticipated it except in the most general terms." 25 Shimony proceeds to argue that "the discrepancies ... between Whiteheadian physics and current microphysics constitute strong disconfirmation of Whitehead's philosophy as a whole."26 And Henry Folse argues, conversely, that "the philosophy of organism provides a natural context for the acceptance of the Copenhagen Interpretation of quantum theory, especially with the ideas of Bohr and Heisenberg." ${ }^{27}$ Folse goes on to say:

Quite naturally there are many aspects of the philosophy of organism which find no counterpart in the philosophical extrapolations of the Copenhagen Interpretation. ... There is no reference to the equivalents of "feeling," "satisfaction," or "conceptual prehension." Yet Whitehead would have anticipated this, for the physicists' interpretation of theory is based on a very small segment of experience; Whitehead's system aims at far greater compass....

The Copenhagen position has come under considerable criticism in recent years, much of which draws its strength upon an appeal to 
the classical ontology of mechanistic materialism. It would seem that the Copenhagen Interpretation and process philosophy would make good allies in any battle against resurgent substantival materialism. However, the fate of any potential alliance is in jeopardy so long as current discussions of the subject insist on concentrating on the fine points of quantum interpretation rather than its broader more general ramifications. ${ }^{28}$

These points are well taken; however, it has been the purpose of this introductory section to demonstrate that Whitehead did indeed anticipate that the quantum theory would be an exemplification of his cosmological scheme, and not merely vaguely compatible with it. Indeed, to counter Folse's first point above, Whitehead goes so far as to suggest specific correlations between the nomenclature of his scheme and that of quantum theory:

If we substitute the term "energy" for the concept of a quantitative emotional intensity, and the term "form of energy" for the concept of "specific form of feeling," and remember that in physics "vector" means definite transmission from elsewhere, we see that this metaphysical description of the simplest elements in the constitution of actual entities agrees absolutely with the general principles according to which the notions of modern physics are framed. The "datum" in metaphysics is the basis of the vector-theory in physics; the quantitative satisfaction in metaphysics is the basis of the scalar localization of energy in physics; the "sensa" in metaphysics are the basis of the diversity of specific forms under which energy clothes itself. .. The general principles of physics are exactly what we should expect as a specific exemplification of the metaphysics required by the philosophy of organism. ${ }^{29}$ (emphasis added)

Whitehead's claim here should not be overlooked; the metaphysical scheme he presents in Process and Reality and in other writings was absolutely intended to be a fundamental characterization of nature as exemplified by the theoretical physics of his time, which included the development of modern quantum mechanics. Aside from his explicitly saying so, the overwhelming detail in which he presents his cosmological model is more than indicative of that intention. For how could it be that the fundamental features of his philosophy are reflected and analyzable in practically every aspect of human experience, as thoroughly elaborated upon in his writings, except that of the physics of his time? If any aspect of human experience closely 
correlates with the specific features of Whitehead's metaphysical scheme, it should certainly be the latter-especially given that the quantum theory is a purely mathematical theory, and therefore clearly within the technical scope of Whitehead's expertise.

It is the purpose of this chapter to demonstrate how quantum mechanics, as given by the modern decoherence-based interpretations described thus far ${ }^{30}$ is, in the most specific terms of the mechanics, an extremely precise, phase-by-phase exemplification of Whitehead's cosmological model. It is an exemplification both conceptually and mechanically, and in terms of physical nature, quantum mechanics is thus the most fundamental exemplification of Whiteheadian metaphysics currently capable of analysis.

The most general correlation between quantum mechanics and the Whiteheadian cosmological system pertains to the concept of state evolution in the former, and concrescence in the latter. These terms describe the same process as elaborated in Part I, wherein:

(i) A world of existing, mutually interrelated facts (Whitehead's "actual occasions") is presupposed.

(ii) The inclusion of these facts (Whitehead's "positive prehension" or "feeling" of facts as "data") in the act of measurement or state specification of them-by their necessary mutual interrelations, somehow entails:

(a) All other facts and their associated potentia-either in their inclusion in the specification, or their necessary exclusion from specification. This requirement is reflected in Whitehead's "Principle of Relativity" and his "Ontological Principle," and in quantum mechanics, by the Schrödinger equation's exclusive applicability to closed systems, with the universe being the only such system. ${ }^{31}$ The exclusions relate to the process of negative selection productive of the decoherence effect described earlier, and Whitehead refers to these eliminations as "negative prehensions." Their form and function with respect to environmental degrees of freedom are, as we shall see, identical to those related to the process of decoherence.

(b) The evolution of the system of all facts into a novel factnamely, a maximal specification (the "state" specification) of the relevant facts (those not excluded by decoherence or "negatively prehended" in Whitehead's terminology). State specification-the maximal specification of many facts via 
the necessary exclusion of some facts-thus entails the evolutionary production of a novel fact-namely, a unification of the facts specified.

(c) The requirement that this evolution proceed relative to a particular fact, typically belonging to a particular subsystem of facts. In quantum mechanics, these are referred to, respectively, as the "indexical eventuality" and the "measuring apparatus"; Whitehead's equivalent term is, simply, the prehending "subject." This requirement is given in Whitehead's "Ontological Principle" and "Category of Subjective Unity"; their correlates in quantum mechanics-the necessary relation of a state evolution to some "preferred basis" characteristic of the measuring apparatus-has often been misapprehended as a principle of sheer subjectivity, the source of the familiar lamentations that quantum mechanics destroys the objective reality of the world.

(iii) Measurement or state specification thus entails, at its heart, the anticipated actualization (or "concrescence") of one novel potential fact/entity from many valuated potential facts/entities which themselves arise from antecedent facts (data); and it is understood that the quantum mechanical description of this evolution terminates in a matrix of probability valuations, anticipative of a final unitary reduction to a single actuality. Ultimately, then, concrescence/state evolution is unitary evolution from actualities to unique actuality. But when analyzed into subphases, both concrescence and state evolution entail a fundamental nonunitary evolution, analogous to von Neumann's conception of quantum mechanics as most fundamentally a nonunitary state reduction productive of a unitary reduction. ${ }^{32}$ It is an evolution from:

(a) a multiplicity of the actual many-that is, $|\Psi\rangle$, to

(b) a matrix of potential "formal" (in the sense of applying a "form" to the facts) integrations or unifications of the many (Whitehead's term is propositional "transmutations" of the many-a specialized kind of "subjective form" — and he also groups these into "matrices" 33 ). Each of these potential integrations is described in quantum mechanics as a projection of a vector representing the actual, evolving multiplicity of facts onto a vector (or subspace) representing a potential "formally integrated" eigenstate. The Whiteheadian analog of the actual multiplicity's "projection" onto a potential integration is "ingression"-where a potential formal integration 
arises from the ingression of a specific "potentiality of definiteness" 34 via a "conceptual prehension" of that specific potentiality of definiteness (Whitehead also refers to these potentia as "eternal objects," and explicitly equates the two terms $\left.{ }^{35}\right)$. But whereas in quantum mechanics, the state vector representing the actual multiplicity of facts is projected onto the potential integration (the eigenvector representing the eigenstate), in Whitehead's scheme it is the latter which ingresses into the prehensions of the actual multiplicity. This difference reflects Whitehead's concern with the origin of these potentia; according to his Ontological Principle, if they ingress into the evolution, they must be thought of as coming from somewhere. The eigenstate, or object of projection in quantum mechanics, is, in contrast, simply extant. This difference aside, Whiteheadian vector "ingression" and quantum mechanical vector "projection" are conceptually equivalent-as are the terms "eternal object" and "potential fact."

There are, furthermore, two important characteristics shared by both the quantum mechanical and Whiteheadian notions of potentia. First, there is a sense in which both are "pure" potentia, referent to no specific actualities. For Whitehead, "eternal objects are the pure potentials of the universe; and the actual entities differ from each other in their realization of potentials." 36 "An eternal object is always a potentiality for actual entities; but in itself, as conceptually felt, it is neutral as to the fact of its physical ingression in any particular actual entity of the temporal world." ${ }^{37}$ In quantum mechanics, this pure potentiality is reflected in the fact that the state vector $|\Psi\rangle$ can be expressed as the sum of an infinite number of vectors belonging to an infinite number of subspaces in an infinite number dimensions, representing an infinite number of potential states or "potentialities of definiteness," referent to no specific actualities and potentially referent to all. Many of these are incapable of integration, forming nonsensical, interfering superpositions, and are eliminated as negative prehensions in a subsequent phase of concrescence.

Second, quantum mechanical projections are also "inherited" from the facts constituting the initial state of the system (as well as the historical route of all antecedent states subsumed by the initial state) such that preferred bases in quantum mechanics are typically 
reproduced in the evolution from state to state. Similarly, in Whitehead's scheme, antecedent facts, when prehended, are typically "objectified" by one of their own historical "potential forms of definiteness" - usually the potential forms that were antecedently actualized at some point in the historical route of occasions constituting the system measured.

An actual entity arises from decisions for it and by its very existence provides decisions for other actual entities which supersede it. ${ }^{38}$

Some conformation is necessary as a basis of vector transition, whereby the past is synthesized with the present. The one eternal object in its two-way function, as a determinant of the datum and as a determinant of the subjective form, is thus relational. . . . An eternal object when it has ingression through its function of objectifying the actual world, so as to present the datum for prehension, is functioning "datively." 39

Whitehead's characterization of potentia as "relational" is exemplified by the manner in which potentia mediate the actuality of a measured system and the actuality of the outcome of the measurement-that is, the mediation of the initial and final system states.

The evolution thus continues into its next phase:

(c) A reintegration of these integrations into a matrix of "qualified propositional" transmutations, ${ }^{40}$ involving a process of negative selection where "negative prehensions" of potentia incapable of further integration are eliminated. The potential unifications or propositional transmutations in this reduced matrix are each qualified by various valuations. Each potential transmutation relative to the indexical eventuality of the measuring apparatus (i.e., each potential outcome state relative to the apparatus and some prehending subject belonging to it) is thus a potential "form" into which the potential facts will ultimately evolve. Whitehead terms these "subjective forms" and as applied to quantum mechanics, the term "subjective" refers to the fact that the "form" of each potential outcome state is reflected in the preferred basis relative to the indexical eventuality of the measuring apparatus (i.e., the prehending subject). Again, it is only the form that is thus subjective-for any number of different devices with different preferred bases could be used to measure a given system, and any number of different people with their own "mental preferred bases" could interpret (measure) the different read- 
ings of the different devices, and so on down the von Neumann chain of actualizations. The potential facts to which each subjective form pertains, however, are initially "given" by the objective facts constitutive of the world antecedent to the concrescence at hand. Thus, again, the "subjective form" of a preferred basis is in no way demonstrative of sheer subjectivity - that is, the evolution of novel facts as determined by a particular subject; it is, rather, demonstrative of the evolution of novel facts jointly determined by both the world of facts antecedent to the evolution and the character of the subject prehending this evolution by virtue of its inclusion in it. Again, according to Whitehead's "Ontological Principle,"

Every condition to which the process of becoming conforms in any particular instance, has its reason either in the character of some actual entity in the actual world of that concrescence, or in the character of the subject which is in process of concrescence. ${ }^{41}$

The actual world is the "objective content" of each new creation. ${ }^{42}$

The evolution thus proceeds to and terminates with what Whitehead terms the "satisfaction" which in quantum mechanical terms is described as:

(d) The actualization of the final outcome state-that is, one subjective form from the reduced matrix of many subjective forms - in "satisfaction" of the probability valuations of the potential outcome states in the reduced matrix. In quantum mechanics, as in Whitehead's model, this actualization is irrelevant and transparent apart from its function as a datum (fact) in a subsequent measurement, such that the "prehending subject" becomes "prehended superject." Again, this is simply because both Whitehead's process of concrescence and quantum mechanics presuppose the existence of facts, and thus cannot account for them. For Whitehead, "satisfaction" entails "the notion of the 'entity as concrete' abstracted from the 'process of concrescence'; it is the outcome separated from the process, thereby losing the actuality of the atomic entity, which is both process and outcome." 43 Thus, the probability valuations of quantum mechanics describe probabilities that a given potential outcome state will be actual upon observation-thus implying a subsequent evolu- 
tion and an interminable evolution of such evolutions. Every fact or system of facts in quantum mechanics, then, subsumes and implies both an initial state and a final state; there can be no state specification $S$ without reference, implicit or explicit, to $S_{\text {initial }}$ and $S_{\text {final }}$. This is reflected in Whitehead's scheme by referring to the "subject" as the "subject-superject":

The "satisfaction" is the "superject" rather than the "substance" or the "subject." It closes up the entity; and yet is the superject adding its character to the creativity whereby there is a becoming of entities superseding the one in question. . . . ${ }^{44}$

An actual entity is to be conceived as both a subject presiding over its own immediacy of becoming, and a superject which is the atomic creature exercising its function of objective immortality. . . ${ }^{45}$

It is a subject-superject, and neither half of this description can for a moment be lost sight of. .. . ${ }^{46}$

[The superject is that which] adds a determinate condition to the settlement for the future beyond itself. ${ }^{47}$

Thus, the process of concrescence is never terminated by actualization/satisfaction; it is, rather, both begun and concluded with it. The many facts and their associated potentia become one novel state (a novel fact), and are thus increased, historically, by one, so that "the oneness of the universe, and the oneness of each element in the universe, repeat themselves to the crack of doom in the creative advance from creature to creature." 48 "The atomic actualities individually express the genetic unity of the universe. The world expands through recurrent unifications of itself, each, by the addition of itself, automatically recreating the multiplicity anew." 49

\section{The Phases of Quantum Mechanical Concrescence}

The process of concrescence is divisible into an initial stage of many feelings, and a succession of subsequent phases of more complex feelings integrating the earlier simpler feelings, up to the satisfaction which is one complex unity of feeling. This is the "genetic" analysis of the 
satisfaction. The actual entity is seen as a process; there is growth from phase to phase; there are processes of integration and reintegration.

Alfred North Whitehead, Process and Reality

The process of concrescence described here by Whitehead is exemplified by the process of quantum mechanical state evolution, which entails the evolution of a closed system of objectively extant facts (data) correlative to the concrescent evolution of a single subjectfact (the indexical eventuality of the measuring apparatus). This evolution consists of a succession of phases in which manifold potential specifications of each datum are integrated-relative to the measuring apparatus and, particularly, its preferred basis. This integration entails the elimination of incompatible and irrelevant specifications via a process of negative selection, productive of the decoherence effect. The result is a matrix of decoherent, mutually exclusive and exhaustive alternative, probability-valuated integrations or potential system states. Of these, one will become actual fact in satisfaction of these valuations as revealed retrodictively by future measurements in its role as datum for these measurements.

The final phase in the process of concrescence ... is termed the "satisfaction." It is fully determinate (a) as to its genesis, (b) as to its objective character for the transcendent creativity [i.e., as datum for subsequent actualizations], and (c) as to its prehension, positive or negative, of every item in its universe. . . . ${ }^{50}$

The satisfaction is merely the culmination marking the evaporation of all indetermination..$^{51}$

Furthermore, the evolution from phase to phase in a concrescence does not occur in asymmetrically modal "physical time," and this is exemplified in quantum mechanics by the time-independent Schrödinger equation which most fundamentally describes this evolution. Each phase, rather, presupposes the actualization as a quantum whole-further exemplified in quantum mechanics, for example, by the inability to specify "what happens between" one observation and the next. This principle, an infamously mystifying component of quantum mechanics for many, is entirely intuitive when interpreted according to the Whiteheadian scheme: For apart from facts, there is nothing to specify.

This genetic passage from phase to phase is not in physical time: the exactly converse point of view expresses the relationship of concres- 
cence to physical time. The actual entity is the enjoyment of a certain quantum of physical time. But the genetic process is not the temporal succession: such a view is exactly what is denied by the epochal theory of time. Each phase in the genetic process presupposes the entire quantum, and so does each feeling in each phase. . . It can be put shortly by saying, that physical time expresses some features of the growth, but not the growth of the features. ${ }^{52}$

Whitehead divides the process of concrescence into three "stages"; 53 the first two stages, Stage I, the responsive stage or "physical pole," and Stage II, the supplementary stage, or "mental pole," entail the integrations of prehensions (or "concrete facts of relatedness") of antecedent facts, and it is these two stages that find their exemplification in quantum mechanical state reduction. Stage III is termed the "satisfaction," which is the actualization of one of the many potential integrations generated in the first two stages. Stages I and II together consist of three successive phases (see figure 4.1, back end sheet), which are precisely analogous to the three phases of state evolution (the valuation of potentia) in orthodox "Copenhagen" quantum mechanics: (i) initial state, which evolves to become (ii) an integration of potentia subsumed by the pure state density matrix, which evolves to become (iii) a reintegration of potentia subsumed by the mixed state, reduced density matrix. Phases $(b)$ and $(c)$ here, as given in the Copenhagen formalism, entail two concepts also present in Whitehead's Phase 2 and Phase 3 in the supplementary stage, as will be explored presently: (i) the transitional nonunitary state evolution suggested by von Neumann; (ii) the process of negative selection productive of the decoherence effect related to this nonunitary state reduction.

It must be emphasized that these two additional concepts in no way require the modification of the orthodox quantum formalism with any additional features (i.e., linear or nonlinear modifications of the Schrödinger equation as suggested by Wigner, for example, or more recently, as suggested in the Spontaneous Localization interpretation of Ghirardi, Rimini, and Weber). All that is required is the orthodox quantum formalism, but applied universally-in satisfaction of the requirement of ontological coherence and consistencyrather than as conventionally and instrumentally applied only to specific situations incapable of accommodation by classical mechanics or ontology. 
Whitehead continues:

There are three successive phases of feelings, namely, a phase of "conformal" feelings [Phase 1, which belongs to Stage I], one of "conceptual" feelings [Phase 2, which belongs to Stage II], and one of "comparative" feelings [Phase 3, which belongs to Stage II], including "propositional" feelings in this last species... . The two latter phases can be put together as the "supplemental" [stage]..$^{54}$

Phases 1, 2, and 3 and their relationship to Stages I, II, and III, have been diagramed in the Appendix (page 246) of this book in order to facilitate the following discussion. The first phase in Whitehead's scheme-the only phase in Stage I, the "responsive stage" or alternately, the "physical pole"-is termed the "primary phase." It is a "conformal," "responsive" phase, wherein the actual world as a multiplicity of facts is initially related to ("prehended" by) the subject. These facts constitute the whole of the antecedent universe relative to the concrescence-that is, facts comprised by the initial state of a measured system, together with that of its measuring apparatus and environment in accord with the closed-system requirement of the Schrödinger equation. This requirement in the ontological interpretation of quantum mechanics is an exemplification of Whitehead's "Principle of Relativity":

The potentiality for being an element in a real concrescence of many entities into one actuality, is the one general metaphysical character attaching to all entities, actual and non-actual [i.e., actual and potential]. Every item in its universe is involved in each concrescence. In other words, it belongs to the nature of a "being" that it is a potential for every "becoming." This is the "principle of relativity." 55

The principle of universal relativity directly traverses Aristotle's dictum, "(A substance) is not present in a subject." On the contrary, according to this principle an actual entity is present in other actual entities. In fact if we allow for degrees of relevance, and for negligible relevance, we must say that every actual entity is present in every other actual entity. The philosophy of organism is mainly devoted to the task of making clear the notion of "being present in another entity." This phrase is here borrowed from Aristotle: it is not a fortunate phrase, and in subsequent discussion it will be replaced by the term "objectification." 56

According to Whitehead's cosmological scheme as exemplified by the ontological interpretation of quantum mechanics, then, in every 
concrescence (or "actualization of a potential fact," or occasion of "state specification" in a measurement interaction) every fact constitutive of the universe is an initial datum which typically becomes "objectified" by one of its own historical potentia. Often, as in most cases of physical transmission of energy, the objectified potential is the one that was actualized in its own process of concrescence. (Recall from Part I that it is this regular reproduction of antecedent potentia throughout historical routes of facts that contributes to the "classicality" of high-inertia systems.) Objectified data are "positively prehended" in Whitehead's terminology, or equivalently, "felt." Thus, the primary phase is the seat of causal efficacy, via "simple physical feelings," in the process of concrescence.

A simple feeling has the dual character of being the cause's feeling reenacted for the effect as subject. But this transference of feeling effects a partial identification of cause with effect, and not a mere representation of the cause. It is the cumulation of the universe and not a stage play about it. By reason of this duplicity in a simple feeling there is a vector character which transfers the cause into the effect. . . Simple physical feelings embody the reproductive character of nature, and also the objective immortality of the past. ${ }^{57}$

Quantum mechanics echoes this conception of the causal interrelations between past facts and the becoming of novel facts. Recall the quote from Omnès:

Past facts are not absolutely real; they only were real. One can never indicate a past fact by pointing a finger at it and saying "that." One must call for a memory or use a record, a note, a photograph. Nevertheless, the derivation of a unique past is possible because quantum mechanics allows for the existence of memory and records. ... What we observe in reality is always something existing right now, even if we interpret it as a trace of an event in the past, whether it be a crater on the moon, the composition of a star atmosphere, or the compared amounts of uranium and lead in a rock. ${ }^{58}$

And in the same spirit, Charles Hartshorne comments, "It is quantum theory that has at last brought science to admit the contingency that qualifies every instance of becoming." 59

In summary of the primary phase of concrescence, Whitehead writes:

The primary stage in the concrescence of an actual entity is the way in which the antecedent universe enters into the constitution of the 
entity in question, so as to constitute the basis of its nascent individuality. .. . ${ }^{60}$

The first phase is the phase of pure reception of the actual world in its guise of objective datum for aesthetic synthesis. ... ${ }^{61}$

The actual world is the "objective content" of each new creation. ${ }^{62}$

The second and third phases of concrescence in Whitehead's scheme together constitute the "supplementary stage" or alternatively, the "mental pole," 63 and whereas the primary stage/physical pole is the seat of causal efficacy, the supplementary stage/mental pole is the seat of causal indeterminism, via "conceptual prehensions" or prehensions of potentia, qualified as two types, "real potentiality" and "general potentiality" (also referred to as "pure potentiality"):

Thus we have always to consider two meanings of potentiality: (a) the "general" potentiality, which is the bundle of possibilities, mutually consistent or alternative, provided by the multiplicity of eternal objects, and (b) the "real" potentiality, which is conditioned by the data provided by the actual world. General potentiality is absolute, and real potentiality is relative to some actual entity, taken as a standpoint whereby the actual world is defined. ${ }^{64}$

A "real potentiality" is potentiality conditioned by the extant facts of the universe to which they pertain-that is, potential facts historically embodied and thus conditioned by the actual universe from which they have evolved. The potential outcome of a measurement of the color status of our red-green traffic signal from Part I, for example, is conditioned by the inheritance of antecedent system states embodied by the history of the traffic-signal system, correlated with the histories of the measuring apparatus and the environment englobing both-that is, the history describing the universe itself. The two "real potentia" of this particular measurement are, then, "red light" and "green light" (assuming these are the only two potential colors to ever have been actualized throughout the history of that traffic signal). It is via such "real potentia" that the causal prehensions from the primary phase are objectified as "immanent," historically realized determinants of the facts prehended. But in the supplementary stage of the currently evolving concrescence, these potentia are now also transcendent capacities for determination; for if our traffic signal were determined via measurement to be red just prior to the current measurement, the outcome might nevertheless be green this time. 
Each entity in the universe of a given concrescence can, so far as its own nature is concerned, be implicated in that concrescence in one or other of many modes; but in fact it is implicated only in one mode. ... The particular mode of implication is only rendered fully determinate by that concrescence, though it is conditioned by the correlate universe. This indetermination, rendered determinate in the real concrescence, is the meaning of "potentiality." It is a conditioned indetermination, and is therefore called a "real potentiality." 65

Real potentia so defined reflect Whitehead's "Category of Conceptual Reproduction"-one of his nine "Categoreal Obligations," which are necessarily presupposed by the process of concrescence, as well as by the ontological interpretation of quantum mechanics described here. The Category of Conceptual Reproduction is described by Whitehead thus:

From each physical feeling there is the derivation of a purely conceptual feeling whose datum is the eternal object exemplified in the definiteness of the actual entity, or the nexus, physically felt. ${ }^{66}$

Conceptual Reproduction (or equivalently, "Conceptual Valuation") constitutes the first subphase of Phase 2-namely, the beginning of the supplementary stage/mental pole. And whereas the conditioned indetermination of "real potentia" in this first subphase provides conditional transcendent novelty to an evolving fact, the unconditioned indetermination of "pure potentia" provides unconditioned transcendent novelty. A "pure potentiality" (or "eternal object")_a "pure potential for the specific determination of fact" 67 - "does not involve a necessary reference to any definite actual entities of the temporal world. . . An eternal object is always a potentiality for actual entities; but in itself, as conceptually felt, it is neutral as to the fact of its physical ingression in any particular actual entity of the temporal world." 68

An eternal object can be described only in terms of its potentiality for "ingression" into the becoming of actual entities; and that its analysis only discloses other eternal objects. It is a pure potential. The term "ingression" refers to that particular mode in which the potentiality of an eternal object is realized in a particular actual entity, contributing to the definiteness of that actual entity. ${ }^{69}$

The unconditioned indetermination provided by pure potentia, like the conditioned indetermination of real potentia, reflects an- 
other Categoreal Obligation: the "Category of Conceptual Reversion" which occurs in the second subphase of Phase 2:

There is a secondary origination of conceptual feelings with data which are partially identical with, and partially diverse from, the eternal objects forming the data in the first phase of the mental pole. . .

Thus the first phase of the mental pole is conceptual reproduction, and the second phase is a phase of conceptual reversion. ... It is the category by which novelty enters the world; so that even amid stability there is never undifferentiated endurance. But, as the category states, reversion is always limited by the necessary inclusion of elements identical with elements in feelings of the antecedent phase. ${ }^{70}$

Note that [the Category of Conceptual Reproduction] concerns conceptual reproduction of physical feeling, and [the Category of Conceptual Reversion] concerns conceptual diversity from physical feeling. ${ }^{71}$

In Whitehead's metaphysical scheme and in quantum mechanics, alternative potential integrations of data (alternative specifications of facts belonging to the system measured) - that is, alternative "forms" of specified factual relations (prehensions of data) - are always relative to a single evolving fact (the prehending subject/indexical eventuality of the measuring apparatus); and thus, these alternative forms of integration-to use Whitehead's term-are "subjective forms." The subjective forms of both real and pure potentia are "valuations"- that is, they have a qualified intensity in a given concrescence-"valuation up" ("adversion") or "valuation down" ("aversion"). ${ }^{72}$ Thus, the subjective form of a "real" potential-that is, a Conceptual Reproduction-is either valuated "up" or "down" compared to other alternative subjective forms of alternative real potentia, which are similarly valuated. Subjective forms of Conceptual Reversions are also valuated "up" or "down."

In quantum mechanics, these valuations are exemplified as probability valuations by which each potential outcome state (i.e., each alternative potential integration of specified facts belonging to a measured system together with its apparatus) is qualified. Thus, the subjective form "traffic signal = green" is a potential integration of facts belonging to the traffic signal, unified according to a "real" potential (light $=$ green) previously actualized in the historical trafficsignal system. "Traffic signal = green" is, then, a Conceptual Reproduction. In quantum mechanics, this subjective form (potential out- 
come state) is valuated "up" or "down" according to a probability between zero and one. And similarly, the subjective form "traffic signal = blue" is a potential integration of facts belonging to the traffic signal, unified according to a "pure" potential not previously actualized in the historical traffic signal system. "Traffic signal = blue," then, is a Conceptual Reversion. In quantum mechanics, even the subjective form of a conceptual reversion is valuated "up" or "down" according to a probability between zero and one. Recall the calculation Murray Gell-Mann described making as an undergraduate wherein he predicted the probability that a heavy macroscopic object would leap a foot into the air of its own accord; the subjective form of that particular Conceptual Reversion was valuated as a probability of $1 / 1 \times 10^{62}$-most definitely an "aversion" or "valuation down" in relation to the subjective forms of the Conceptual Reproductions pertaining to that calculation, one of which was "the object remains at rest."

This distinction between pure and real potentia, including their respective roles in the first two subphases of the supplementary stage-Conceptual Reproduction and Conceptual Reversion-have precise analogs in quantum mechanics; in fact, the representation of potentia as vectors in Hilbert space provides an excellent model in aid of understanding the functional relationship between real and pure potentia in Whitehead's metaphysical scheme. Recall that our idealized red-green traffic signal system was described via a Hilbert space of two dimensions, represented by $x-y$ coordinate axes, such that a vector of unit length along each axis represents the state of the system $S$ as $S_{\text {green }}$ or $S_{\text {red, }}$, respectively. $S_{\text {green }}$ and $S_{\text {red }}$ are thus "real potentia" of the system. One may even generalize that it is a system's "real potentia," correlated with those of the indexical eventuality of the measuring apparatus, which determine the number of mutually orthogonal vectors-and therefore the number of dimensionsrequired by a Hilbert space representing the system. Recall that mutually orthogonal vectors represent mutually exclusive potential states-a requirement of Whiteheadian potentia: "The definiteness of the actual arises from the exclusiveness of eternal objects in their function as determinants. If the actual entity be this, then by the nature of the case it is not that or that. The fact of incompatible alternatives is the ultimate fact in virtue of which there is definite character." 73 
These two sentences together convey the very essence of quantum mechanics. We have already seen their mathematical exemplification: If the mutually orthogonal vectors $\left|u_{\text {green }}\right\rangle$ and $\left|u_{\text {red }}\right\rangle$ represent "real" mutually exclusive potential states of the traffic signal system, then $|\psi\rangle$ —a vector of unit length which belongs to neither the subspace $\mathscr{E}_{\text {green }}$ nor $\mathscr{E}_{\text {red }}$-represents the potential ingression of all pure potentia. For $|\psi\rangle$ can be expressed as the sum of a practically infinite number of vectors, each representing a purely potential state, referent to no particular facts or history of facts; thus some of these purely potential states are sensible and capable of integration in the current evolution, and some are not. Those which are must be mutually exclusive according to both quantum mechanics and Whitehead's cosmological scheme. These are the "real potentia"-in this case, $\left|u_{\text {green }}\right\rangle$ and $\left|u_{\text {red }}\right\rangle$, which in terms of the pure potentiality inherent in $|\psi\rangle$, are expressed:

$$
|\psi\rangle=\alpha\left|u_{\text {green }}\right\rangle+\beta\left|u_{\text {red }}\right\rangle
$$

where $|\alpha|^{2}+|\beta|^{2}=1$.

One of these real potentia $\left|u_{\text {green }}\right\rangle$ or $\left|u_{\text {red }}\right\rangle$ will become actual, according to its valuation as expressed by the complex coefficient $\alpha$ or $\beta$. Both $\left|u_{\text {green }}\right\rangle$ and $\left|u_{\text {red }}\right\rangle$, as real potentia, are (i) realized determinants of facts historically actualized, and in that sense, are "immanent"; and (ii) capacities for determination in the current evolution, and in this sense, "transcendent." 74

The representation of pure potentia (Whitehead's "eternal objects") in the expression $|\psi\rangle=\alpha\left|u_{\text {green }}\right\rangle+\beta\left|u_{\text {red }}\right\rangle$ is disclosed when it is recalled that $\alpha$ and $\beta$-the valuations of the real potentials $\left|u_{\text {green }}\right\rangle$ and $\left|u_{\text {red }}\right\rangle$ respectively-are projections of $|\psi\rangle$ onto $\left|u_{\text {green }}\right\rangle$ and $\left|u_{\text {red }}\right\rangle$ respectively. In Whiteheadian terms, this is the ingression of both the real potentia $\left|u_{\text {green }}\right\rangle$ and $\left|u_{\text {red }}\right\rangle$ into the concrescence, along with the other pure potential states embodied by $|\psi\rangle$ (again, represented mathematically by its potential expression as the sum of a practically infinite number of vectors). Recall that the state vector $|\psi\rangle$, referred to as a "pure state" in quantum mechanics, represents a pure potential referent at once to no specific facts and to all facts. Yet it is an objectively real state, for it is also a maximal specification of the bare multiplicity of facts constituting the system in its initial state-that is, facts determined antecedent to the concrescence at hand. Thus, 
the expression $|\psi\rangle=\alpha\left|u_{\text {green }}\right\rangle+\beta\left|u_{\text {red }}\right\rangle$ represents the dipolar nature of the concrescence given in both the primary stage of conditioned causal determination, and in the supplementary stage of conditioned indetermination.

The process of becoming is dipolar, (i) by reason of its qualification by the determinateness of the actual world, and (ii) by its conceptual prehensions of the indeterminateness of eternal objects. The process is constituted by the influx of eternal objects into a novel determinateness of feeling which absorbs the actual world into a novel actuality. ${ }^{75}$

Clearly, however, $|\psi\rangle$, given its component of pure potentiality, can be expressed as the sum of a multiplicity of vectors and projections other than those contained in the integration $\alpha\left|u_{\text {green }}\right\rangle+\beta\left|u_{\text {red }}\right\rangle$. These coherent superpositions of potentia are eliminated, and it is this elimination which, in a subsequent phase, will produce the two decoherent, mutually exclusive and exhaustive real potential outcome states represented by $\alpha\left|u_{\text {green }}\right\rangle+\beta\left|u_{\text {red }}\right\rangle$. The Whiteheadian account of this elimination and its mechanism will be discussed presently, but for now can be inferred from the two sentences cited above: "The fact of incompatible alternatives is the ultimate fact in virtue of which there is definite character." Or, in the words of Hartshorne, "An actual world cannot be all possible worlds. . . To be actual is to exclude some possibilities." 76

The initial problem is to discover the principles according to which some eternal objects are prehended positively and others are prehended negatively. Some are felt and others are eliminated. ${ }^{77}$

Before exploring the concept and mechanism of these eliminations in the form of negative prehensions, we must provide a more accurate mathematical expression for the process of concrescence thus far; for $|\psi\rangle=\alpha\left|u_{\text {green }}\right\rangle+\beta\left|u_{\text {red }}\right\rangle$ only represents the integrations of prehensions. The concrescing subject-superject (i.e., the indexical eventuality belonging to a measuring apparatus) is only implied. All that is required is an expression which correlates the concrescent evolution of the measured (prehended) system with that of the measuring apparatus, relative to the indexical eventuality/prehending subject. The following expression, introduced and discussed at length in Part I, does this: 


$$
\left|\Phi^{c}\right\rangle=\alpha\left|u_{\text {green }}\right\rangle\left|d_{\downarrow}\right\rangle+\beta\left|u_{\text {red }}\right\rangle\left|d_{\uparrow}\right\rangle
$$

But as we have seen, the elimination of superpositions of incompatible potentia in quantum mechanics requires the correlation of facts belonging to the evolving system-apparatus with those belonging to the whole of the environment which englobes them. These environmental correlations are also required by any coherent ontological interpretation of quantum mechanics, which applies only to closed systems such as that of a universe characterized as systemapparatus-environment. Whitehead holds the same requirement in his metaphysical scheme, where the concrescent evolution of any one potential fact or system ("nexus") of such facts necessarily entails all facts antecedent to the evolution. And as is the case in quantum mechanics, Whitehead's elimination via negative prehensions of incompatible potentia-the "thwarting elements of a nexus" 78 occurs by virtue of the elimination of the "unwelcome detail"79 generated by these necessary environmental correlations-precisely the same mechanism described by the decoherence-based interpretations of quantum mechanics discussed in Part I. We can now proceed to explore in more detail these conceptual similarities between Whitehead's process of negative selection and that of the decoherence effect in quantum mechanics, as the environmental correlations required by both are accounted for in the expression:

$$
\begin{aligned}
\left|\Phi^{c}\right\rangle\left|E_{0}\right\rangle & =\left(\alpha\left|u_{\text {green }}\right\rangle\left|d_{\downarrow}\right\rangle+\beta\left|u_{\text {red }}\right\rangle\left|d_{\uparrow}\right\rangle\right)\left|E_{0}\right\rangle \\
& \Rightarrow \alpha\left|u_{\text {green }}\right\rangle\left|d_{\downarrow}\right\rangle\left|E_{\downarrow}\right\rangle+\beta\left|u_{\text {red }}\right\rangle\left|d_{\uparrow}\right\rangle\left|E_{\uparrow}\right\rangle=|\Psi\rangle
\end{aligned}
$$

where $|\Psi\rangle$ is the system (nexus) of all facts prehended as two "real," and therefore mutually exclusive, ${ }^{80}$ potential integrations $\alpha\left|u_{\text {green }}\right\rangle\left|d_{\downarrow}\right\rangle\left|E_{\downarrow}\right\rangle$ and $\beta\left|u_{\text {red }}\right\rangle\left|d_{\uparrow}\right\rangle\left|E_{\uparrow}\right\rangle$, each one a valuated "subjective form" of $|\Psi\rangle$ that is, an integration relative to the prehending subject/indexical eventuality $|d\rangle$ belonging to the detector. Thus, we have a mathematical expression of the process of concrescence (excluding the "satisfaction"),

$$
|\Psi\rangle=\alpha\left|u_{\text {green }}\right\rangle\left|d_{\downarrow}\right\rangle\left|E_{\downarrow}\right\rangle+\beta\left|u_{\text {red }}\right\rangle\left|d_{\uparrow}\right\rangle\left|E_{\uparrow}\right\rangle
$$

which describes

a growth of prehensions, with integrations, eliminations, and determination of subjective forms. But the determination of successive phases of subjective forms, whereby the integrations have the characters that 
they do have, depends on the unity of the subject imposing a mutual sensitivity upon the prehensions. ${ }^{81}$

This equation is conceptually similar to that describing pure state discussed above-

$$
|\psi\rangle=\alpha\left|u_{\text {green }}\right\rangle+\beta\left|u_{\text {red }}\right\rangle
$$

- only now correlated with facts belonging to both the detector and environment, in satisfaction of the requirements of ontological coherence.

\section{The Categoreal Obligations}

Five Whiteheadian "Categoreal Obligations" are operative thus far in our examination of the quantum mechanical exemplification of concrescence-two of which we have already discussed (the "Category of Conceptual Reproduction" and the "Category of Conceptual Reversion") - and it will be useful to describe the other three here prior to discussing the process of negative selection, which will introduce several others. It is important to note that these Categoreal Obligations are not only exemplified in quantum mechanics; they are presupposed by quantum mechanics. The first three pertain to the primary stage of concrescence.

\section{Categoreal Obligation I: The Category of Subjective Unity}

The many feelings which belong to an incomplete phase in the process of an actual entity, though unintegrated by reason of the incompleteness of the phase, are compatible for synthesis by reason of the unity of their subject. ${ }^{82}$

Quantum mechanics describes the evolution of the state of a system as a set of alternative integrations of potential facts born of antecedent "actual" facts, and these integrations are always relative to a particular fact-the indexical eventuality-as it also evolves. It is this state evolution relative to a single evolving eventuality and its preferred basis which allows these integrations to occur; and it is by virtue of this relativity that all potentia involved in the evolution will be compatible, and that those which are not will be eliminated. 
The novel actual entity, which is the effect, is the reproduction of the many actual entities of the past. But in this reproduction there is abstraction from their various totalities of feeling. This abstraction is required by the categoreal conditions for compatible synthesis in the novel unity. The limitation, whereby the actual entities felt are severally reduced to the perspective of one of their own feelings, is imposed by the categoreal condition of subjective unity, requiring a harmonious compatibility in the feelings of each incomplete phase. This subjective insistence may, from the beginning, replace positive feelings by negative prehensions. Feelings are dismissed by negative prehensions, owing to their lack of compliance with categoreal demands. ${ }^{83}$

\section{Categoreal Obligation II: The Category of Objective Identity}

There can be no duplication of any element in the objective datum of an actual entity, so far as concerns the function of that element in the satisfaction. ... This category asserts the essential self-identity of any entity as regards its status in each individualization of the universe. In such a concrescence one thing has one rôle, and cannot assume any duplicity. This is the very meaning of self-identity, that, in any actual confrontation of thing with thing, one thing cannot confront itself in alien rôles. Any one thing remains obstinately itself playing a part with self-consistent unity. This category is one ground of incompatibility. ${ }^{84}$

The elimination of superpositions of interfering potentia in quantum mechanics is an exemplification of this category, which obliges the satisfaction of the logical principle of noncontradiction. Simply, it is by the Category of Objective Identity that a confused creature like Schrödinger's cat cannot exist objectively in its superposed state.

\section{Categoreal Obligation III: The Category of Objective Diversity}

There can be no "coalescence" of diverse elements in the objective datum of an actual entity, so far as concerns the functions of those elements in that satisfaction. . . "Coalescence" here means the notion of diverse elements exercising an absolute identity of function, devoid of the contrasts inherent in their diversities. ${ }^{85}$

The third category is concerned with the antithesis to oneness, namely, diversity. An actual entity is not merely one; it is also definitely complex. But, to be definitely complex is to include definite diverse elements in definite ways. The category of objective diversity expresses the inexorable condition-that a complex unity must pro- 
vide for each of its components a real diversity of status, with a reality which bears the same sense as its own reality and is peculiar to itself. In other words, a real unity cannot provide sham diversities of status for its diverse components. ${ }^{86}$

Category III is particularly important to any ontological interpretation of quantum mechanics which characterizes potentia as a species of reality rather than merely an artifact of some epistemic boundary that cannot be crossed. For in an interpretation which maintains the latter, the quantum mechanical evolution of a system is fundamentally a system of subatomic, material locomotions. What evolves is merely our knowledge of these locomotions, and therefore the diverse potentia "integrated" in the evolution are "sham diversities of status"-vacuous, statistical, necessarily approximate specifications of these locomotions, where the "status" of one such potential is equivalent to the status of any.

In the interpretation of quantum mechanics described here, however, the ontological significance of potentia presupposes Category III; for if potentia are indeed a species of reality, then each potential must be as unique as the specific actuality from which it evolves. And therefore integrations of potentia are "real diversities."

Because Category IV, the Category of Conceptual Reproduction, and Category V, the Category of Conceptual Reversion were already introduced above, we can now move on to Category VI.

\section{Categoreal Obligation VI: The Category of Transmutation}

Returning to our idealized traffic signal system, the quantum mechanical expression of concrescence is given as:

$$
|\Psi\rangle=\alpha\left|u_{\text {green }}\right\rangle\left|d_{\downarrow}\right\rangle\left|E_{\downarrow}\right\rangle+\beta\left|u_{\text {red }}\right\rangle\left|d_{\uparrow}\right\rangle\left|E_{\uparrow}\right\rangle
$$

This expression, in both the decoherence-based interpretations of quantum mechanics and in Whitehead's scheme, presupposes a process of negative selection via negative prehensions. This process, as we saw in Part I, is expressed via the use of matrices of potentia-or more precisely, matrices of alternative integrations of potentia. These integrations are exemplifications of the Whiteheadian "subjective forms" of potentia, given that these potentia are expressed in terms of $|d\rangle$-that is, relative to $|d\rangle$ as the prehending subject/indexical eventuality belonging to the detector. The pure 
state density matrix contains all the ingressed potentia, pure and real, related to the data of the correlated system-apparatus-environment.

$$
\begin{aligned}
\rho^{c} & =|\Psi\rangle\langle\Psi| \\
& =|\alpha|^{2}\left|u_{\text {green }}\right\rangle\left\langle u_{\text {green }} \| d_{\downarrow}\right\rangle\left\langle d_{\downarrow} \| E_{\downarrow}\right\rangle\left\langle E_{\downarrow}\right| \\
& +\boldsymbol{\alpha} \boldsymbol{\beta}\left|u_{\text {green }}\right\rangle\left\langle\boldsymbol{u}_{\text {red }} \| \boldsymbol{d}_{\downarrow}\right\rangle\left\langle\boldsymbol{d}_{\uparrow} \| \boldsymbol{E}_{\downarrow}\right\rangle\left\langle\boldsymbol{E}_{\uparrow}\right| \\
& +\boldsymbol{\alpha}^{*} \boldsymbol{\beta}\left|\boldsymbol{u}_{\text {red }}\right\rangle\left\langle\boldsymbol{u}_{\text {green }}|| \boldsymbol{d}_{\uparrow}\right\rangle\left\langle\boldsymbol{d}_{\downarrow} \| \boldsymbol{E}_{\uparrow}\right\rangle\left\langle\boldsymbol{E}_{\downarrow}\right| \\
& +|\boldsymbol{\beta}|^{2}\left|u_{\text {red }}\right\rangle\left\langle u_{\text {red }}|| d_{\uparrow}\right\rangle\left\langle d_{\uparrow}|| E_{\uparrow}\right\rangle\left\langle E_{\uparrow}\right|
\end{aligned}
$$

Some of these potentia are capable of integration into the mutually exclusive subjective forms $\alpha\left|u_{\text {green }}\right\rangle\left|d_{\downarrow}\right\rangle\left|E_{\downarrow}\right\rangle$ and $\beta\left|u_{\text {red }}\right\rangle\left|d_{\uparrow}\right\rangle\left|E_{\uparrow}\right\rangle$; and others are incapable of such integration because of their mutual nonexclusivity (interference) and thus constitute coherent superpositions of potentia. These are represented by the two middle terms in boldface and are the nonsensical subjective forms that are eliminated from the concrescing evolution by virtue of the excess prehensions of environmental data they include-again, "excess" relative to the prehending subject/indexical eventuality. These excess potentia are eliminated in the Whiteheadian scheme via

a massive average objectification of a nexus, while eliminating the detailed diversities of the various members of the nexus in question. This method, in fact, employs the device of blocking out unwelcome detail. It depends on the fundamental truth that objectification is abstraction. It utilizes this abstraction inherent in objectification so as to dismiss the thwarting elements of a nexus into negative prehensions. At the same time the complex intensity in the structured society is supported by the massive objectifications of the many environmental nexūs, each in its unity as one nexus, and not in its multiplicity as many actual occasions.

This mode of solution requires the intervention of mentality operating in accordance with the Category of Transmutation. It ignores diversity of detail by overwhelming the nexus by means of some congenial uniformity which pervades it. The environment may then change indefinitely so far as concerns the ignored details—so long as they can be ignored.

The close association of all physical bodies, organic and inorganic alike ... suggests that this development of mentality [i.e., activity of the "Mental Pole" or "Supplementary Phase" — not conscious mental- 
ity] is characteristic of the actual occasions which make up the structured societies we know as "material bodies." 87

The mathematical analog of the Category of Transmutation is, as we have seen, the "trace-over" or "sum-over" of the potentia in the pure state density matrix such that the "detailed diversities" stemming from the environmental correlations, represented by the offdiagonal terms, are eliminated:

$$
\rho_{\mathrm{SD}} \equiv \operatorname{Tr}_{E}|\Psi\rangle\langle\Psi|=\Sigma_{i}\left\langle E_{i} \mid \Psi\right\rangle\left\langle\Psi \mid E_{i}\right\rangle=\mathrm{Q}^{r}
$$

This yields a reduced density matrix $\mathrm{Q}^{r}$ of mutually exclusive and exhaustive subjective forms, or valuated "real" potential integrations of the system-apparatus nexus

$$
\rho^{\mathrm{r}}=|\alpha|^{2}\left|u_{\text {green }}\right\rangle\left\langle u_{\text {green }}|| d_{\downarrow}\right\rangle\left\langle\left. d_{\downarrow}|+| \beta\right|^{2} \mid u_{\text {red }}\right\rangle\left\langle u_{\text {red }}|| d_{\uparrow}\right\rangle\left\langle d_{\uparrow}\right|
$$

and evinces the "Principle of Relativity" so crucial to Whitehead's cosmology: Every fact is a potential determinant in the becoming of every new fact. This reduced density matrix is merely another way of expressing the earlier equation:

$$
\left|\Phi^{\mathrm{c}}\right\rangle=\alpha\left|u_{\text {green }}\right\rangle\left|d_{\downarrow}\right\rangle+\beta\left|u_{\text {red }}\right\rangle\left|d_{\uparrow}\right\rangle
$$

This "massive average objectification" is possible mathematically for the same reason it is possible conceptually in Whitehead's scheme; it is prefaced by an integration of a vast multiplicity of potential outcomes (owing to the manifold degrees of freedom contributed by the environment) into various "subjective forms" relative to the prehending subject/indexical eventuality $|d\rangle$. In the case of our traffic light example, this yields two subjective forms:

(i) $|\alpha|^{2}\left|u_{\text {green }}\right\rangle\left\langle u_{\text {green }}|| d_{\downarrow}\right\rangle\left\langle d_{\downarrow} \| \mid E_{\downarrow}\right\rangle\left\langle E_{\downarrow}\right|$

(ii) $\left.|\beta|\right|^{2}\left|u_{\text {red }}\right\rangle\left\langle u_{\text {red }}|| d_{\uparrow}\right\rangle\left\langle d_{\uparrow}|| E_{\uparrow}\right\rangle\left\langle E_{\uparrow}\right|$

Each subjective form is a potential integration of all facts belonging to the nexus of facts comprising the system, the detector, and the environment relative to the prehending subject/indexical eventuality $|d\rangle$ belonging to the detector. Each potential integration, by the manifold environmental correlations, thus entails a practically infinite number of potentia-some prehended positively, others negatively-each one related to a separate environmental datum. Each of these two subjective forms consists of manifold potential integra- 
tions of these prehensions of the system-apparatus-environment nexus, but each shares a common, "defining characteristic" determined by each subjective form-namely, the particular status of the indexical eventuality $|d\rangle$. In mathematical terminology, each subjective form is thus referred to as an "equivalence class" or "coarsegrained" integration. It is this grouping into equivalence classes or Whiteheadian "subjective forms" that allows for the cancellations among ignored "detailed diversities," thus "blocking out unwelcome detail" (or "coarse graining" these details); and it is by these cancellations that the elimination of the interfering, incompatible potentia, represented by the two terms in boldface on p. 149, is effected.

The irrelevant multiplicity of detail is eliminated, and emphasis is laid on the elements of systematic order in the actual world. ${ }^{88}$

In this process, the negative prehensions which effect the elimination are not merely negligible. The process through which a feeling passes in constituting itself, also records itself in the subjective form of the integral feeling. The negative prehensions have their own subjective forms which they contribute to the process. A feeling bears on itself the scars of its birth; it recollects as a subjective emotion its struggle for existence; it retains the impress of what it might have been, but is not. It is for this reason that what an actual entity has avoided as a datum for feeling may yet be an important part of its equipment. The actual cannot be reduced to mere matter of fact in divorce from the potential. ${ }^{89}$

The right co-ordination of negative prehensions is one secret of mental progress; but unless some systematic scheme of relatedness characterizes the environment, there will be nothing left whereby to constitute vivid prehension of the world. ${ }^{90}$

Again, this "right co-ordination of negative prehensions" via a "systematic scheme of relatedness" characterizing the environment, productive of correlate coordinations of positive prehensions, occurs in Phase 3 of the concrescence in accord with the Category of Transmutation, which "ignores diversity of detail by overwhelming the nexus by means of some congenial uniformity which pervades it. The environment may then change indefinitely so far as concerns the ignored details-so long as they can be ignored." 91 The process of decoherence, as described above-via integrations of potentia into "equivalence classes" or "subjective forms" according to a defining 
characteristic - is a precise exemplification of the Category of Transmutation; for it describes the integration of a bare multiplicity of facts into a potential system (nexus) where each potential fact in the potential system shares a "defining characteristic" given in the indexical eventuality of the measuring apparatus. Thus, each potential integration of this kind (a nexus with "social order") is exemplified mathematically as an "equivalence class" or "coarse-grained" integration:

A "society" . . is a nexus with social order. A nexus enjoys "social order" where (i) there is a common element of form illustrated in the definiteness of each of its included actual entities, and (ii) this common element of form arises in each member of the nexus by reason of the conditions imposed upon it by its prehensions of some other members of the nexus, and (iii) these prehensions impose that condition of reproduction by reason of their inclusion of positive feelings of that common form. Such a nexus is called a "society," and the common form is the "defining characteristic" of the society. The notion of "defining characteristic" is allied to the Aristotelian notion "substantial form."

And as this concept applies to the Category of Transmutation, defined by Whitehead:

When (in accordance with category IV, or with categories IV and V) one, and the same, conceptual feeling is derived impartially by a prehending subject from its analogous simple physical feelings of various actual entities, then in a subsequent phase [Phase 3] of integration-of these simple physical feelings together with the derivate conceptual feeling - the prehending subject may transmute the datum of this conceptual feeling into a characteristic of some nexus containing those prehended actual entities, or of some part of that nexus; so that the nexus (or its part), thus qualified, is the objective datum of a feeling entertained by this prehending subject. Such a transmutation of simple physical feelings of many actualities into one physical feeling of a nexus as one, is called a "transmuted feeling." 93

It is evident that the complete datum of the transmuted feeling is a contrast, namely, "the nexus, as one, in contrast with the eternal object." This type of contrast is one of the meanings of the notion "qualification of physical substance by quality." 94

The decoherence effect in quantum mechanics-via coarsegrained, alternative "equivalence classes" productive of the necessary eliminations of interfering potentia-is an exemplification of 
the Category of Transmutation both conceptually and "mechanically": Both the decoherence effect and the Category of Transmutation play an identical role in accounting for the logically ordered, enduring characteristics of the "classical" macroscopic world, including our notion of "qualification of physical substance by quality"; and each ultimately provides the conceptual and mechanical means by which its fundamental characterization of nature can apply to human experience.

The distinction between "conceptual" and "mechanical" means is important here, for Whitehead had the former in mind with respect to the Category of Transmutation more so than the latter; he was not aware, for example, of the recent quantum mechanical interpretations which use the decoherence effect to mechanically account for the classicality of macroscopic objects. But even though Whitehead was more interested in the Category of Transmutation conceptually as it applies to perception (i.e., its use in accounting for perceptive errors ${ }^{95}$ ), he explicitly refrained from predicating Transmutation upon consciousness:

It is evident that adversion and aversion, and also the Category of Transmutation, only have importance in the case of high-grade organisms. They constitute the first step towards intellectual mentality, though in themselves do not amount to consciousness. ${ }^{96}$

Nevertheless, the application of the Category of Transmutation to even the most rudimentary actual occasions-as is suggested here in its relation to the quantum mechanical decoherence effect-would likely not be objected to by Whitehead, for he explicitly proposes the function of Transmutation-and Reversion-in quantum mechanics. Indeed, it is this fundamental physical role of transmutation from which higher-order mental transmutations ultimately derive:

The physical theory of the structural flow of energy has to do with the transmission of simple physical feelings from individual actuality to individual actuality. Thus some sort of quantum theory in physics, relevant to the existing type of cosmic order, is to be expected. The physical theory of alternative forms of energy, and of the transformation from one form to another form, ultimately depends upon transmission conditioned by some exemplification of the Categories of Transmutation and Reversion. ${ }^{97}$

Apart from transmutation our feeble intellectual operations would fail to penetrate into the dominant characteristics of things. We can only 
understand by discarding. ... Transmutation is the way in which the actual world is felt as a community, and is so felt in virtue of its prevalent order. For it arises by reason of the analogies between the various members of the prehended nexus, and eliminates their differences. ${ }^{98}$

Transmutation in Phase 3 of the concrescence, as exemplified in quantum mechanics by the decoherence effect, results in a reduced density matrix of mutually exclusive, "coarse-grained" subjective forms, each valuated as a probability. Thus, in the case of our idealized traffic signal system, each of the terms in the reduced density matrix

(i) $|\alpha|^{2}\left|u_{\text {green }}\right\rangle\left\langle u_{\text {green }}|| d_{\downarrow}\right\rangle\left\langle d_{\downarrow}\right|$

(ii) $|\beta|^{2}\left|u_{\text {red }}\right\rangle\left\langle u_{\text {red }}|| d_{\uparrow}\right\rangle\left\langle d_{\uparrow}\right|$

represents a subjective form or real potential integration of the nexus of facts comprising the system and detector. Excess environmental correlations have been eliminated, carrying with them the coherent superpositions of incompatible potentia incapable of integration. Again, each subjective form is a potential integration (potential societal nexus) of the traffic signal system/detector facts relative to a particular indexical eventuality/prehending subject, specified by $|d\rangle$.

Transmutation entails the "generic contrast" of (i) the real potential serving as the "defining characteristic" by which a given social nexus ("equivalence class") is integrated (in our example, the defining characteristic would be the indexical eventuality $\left|d_{\uparrow}\right\rangle$ or $\left.\left|d_{\downarrow}\right\rangle\right)$; and (ii) the reality of the system of facts $(|\Psi\rangle)$ being prehended (related) in the concrescence (measurement). In quantum mechanics, this is exemplified by the contrast of (i) one of the alternative "real potential," probable measurement outcome states in the reduced matrix (again, "state" being a maximal specification or form of the facts belonging to a system); and (ii) the system of actual, extant facts as data. It is a contrast, in other words, of potential forms with actual facts. This contrast is, once again, mathematically represented as

$$
|\Psi\rangle=\alpha\left|u_{\text {green }}\right\rangle\left|d_{\downarrow}\right\rangle\left|E_{\downarrow}\right\rangle+\beta\left|u_{\text {red }}\right\rangle\left|d_{\uparrow}\right\rangle\left|E_{\uparrow}\right\rangle
$$

and, in Whitehead's scheme, is productive of "comparative feelings," of which "physical purposes" are one type:

The integration of each simple physical feeling [in Phase 1] with its conceptual counterpart [in Phase 2] produces in a subsequent phase 
[Phase 3] a physical feeling whose subjective form of re-enaction has gained or lost subjective intensity according to the valuation up, or the valuation down, in the conceptual feeling. This is the phase of physical purpose. ${ }^{99}$

In the integral comparative feeling the datum is the contrast of the conceptual datum with the reality of the objectified nexus. The physical feeling [or "Concrete Fact of Relatedness"] is feeling a real fact; the conceptual feeling is valuing an abstract possibility. ... This synthesis of a pure abstraction $\left[\alpha\left|u_{\text {green }}\right\rangle\left|d_{\downarrow}\right\rangle\left|E_{\downarrow}\right\rangle\right.$ or $\left.\beta\left|u_{\text {red }}\right\rangle\left|d_{\uparrow}\right\rangle\left|E_{\uparrow}\right\rangle\right]$ with a real fact $[|\Psi\rangle]$, as in feeling, is a generic contrast. . . .

The constancy of physical purposes explains the persistence of the order of nature. ... [In a] physical purpose, the datum is the generic contrast between the nexus, felt in the physical feeling, and the eternal object valued in the conceptual feeling. This eternal object is also exemplified as the pattern of the nexus. Thus the conceptual valuation now closes in upon the feeling of the nexus as it stands in the generic contrast, exemplifying the valued eternal object. This valuation accorded to the physical feeling endows the transcendent creativity with the character of adversion, or of aversion. The character of adversion secures the reproduction of the physical feeling, as one element in the objectification of the subject beyond itself. Such reproduction may be thwarted by incompatible objectification derived from other feelings. But a physical feeling, whose valuation produces adversion, is thereby an element with some force of persistence into the future beyond its own subject. It is felt and re-enacted down a route of occasions forming an enduring object....

When there is aversion, instead of adversion, the transcendent creativity assumes the character that it inhibits, or attenuates, the objectification of that subject in the guise of that feeling. Thus aversion tends to eliminate one possibility by which the subject may itself be objectified in the future. Thus adversions promote stability; and aversions promote change without any indication of the sort of change. ${ }^{100}$

There are two species of physical purpose: (i) physical purposes due to conceptual reproduction; and (ii) physical purposes due to conceptual reversion. A physical purpose of the first species, such as those facts related to the transfer of energy, merely "receives the physical feelings, confirms their valuations according to the 'order' of that epoch, and transmits by reason of its own objective immortality. Its own flash of autonomous individual experience is negligible." ${ }^{101}$ And it is because of "the origination of reversions in the 
[supplementary stage] . . . that vibration and rhythm have a dominating importance in the physical world"102_a concept clearly exemplified by objective quantum indeterminacy.

Any ontological interpretation of quantum mechanics wherein potentia are characterized as a species of reality must at some point address not only the relationship between these potentia and the actualities from which they evolve, but also the relationship between these potentia and the actualities they evolve to become. Phases 1 and 2, and their correlate Categoreal Obligations, primarily concern the former; Phase 3-the phase of comparative feelings and transmutation-is primarily concerned with the latter. It concerns not only what these potential and probable outcome states are in quantum mechanics, and from whence they evolved-questions related to Phases 1 and 2; but also why these potential and probable outcome states are what they are. And as Whitehead's metaphysical scheme entails answers to other ontological questions commonly raised with respect to quantum mechanics, such as those explored thus far, it entails an answer to this important question as well.

The answer is to be found in the three final Categoreal Obligations, which pertain to the balance of adversion and aversionreproduction and reversion-regularity and diversity in the process of concrescence. Whitehead suggests that a tendency toward such balance of adversion and aversion permeates all of nature and is qualitatively manifest as "balanced complexity" —and perhaps quantitatively as well, if indeed quantum mechanics is an exemplification of Whitehead's cosmological scheme.

\section{Categoreal Obligation VII: The Category of Subjective Harmony}

The valuations of conceptual feelings are mutually determined by the adaptation of those feelings to be contrasted elements congruent with the subjective aim. Category I and category VII jointly express a preestablished harmony in the process of concrescence of any one subject. Category I has to do with the data felt, and category VII with the subjective forms of the conceptual feelings. This pre-established harmony is an outcome of the fact that no prehension [or "Concrete Fact of Relatedness"] can be considered in abstraction from its subject, although it originates in the process creative of its subject. ${ }^{103}$

By the Category of Subjective Unity (category I), and by the seventh category of Subjective Harmony, all origination of feelings is governed 
by the subjective imposition of aptitude for final synthesis. In the former category the intrinsic inconsistencies, termed "logical" [i.e., superpositions of nonexclusive, interfering potentia], are the formative conditions in the pre-established harmony. In this seventh category, and in the Category of Reversion, aesthetic adaptation for the end is the formative condition in the pre-established harmony. . . . It is only by reason of the Categories of Subjective Unity, and of Subjective Harmony, that the process constitutes the character of the product, and that conversely the analysis of the product discloses the process. ${ }^{104}$

\section{Categoreal Obligation VIII: The Category of Subjective Intensity}

The subjective aim, whereby there is origination of conceptual feeling, is at intensity of feeling $(\alpha)$ in the immediate subject, and $(\beta)$ in the relevant future. This double aim-at the immediate present and the relevant future-is less divided than appears on the surface. For the determination of the relevant future, and the anticipatory feeling respecting provision for its grade of intensity, are elements affecting the immediate complex of feeling. . . . The relevant future consists of those elements in the anticipated future which are felt with effective intensity by the present subject by reason of the real potentiality for them to be derived from itself. ${ }^{105}$

\section{Categoreal Obligation IX: The Category of Freedom and Determination}

The concrescence of each individual actual entity is internally determined and is externally free... This category can be condensed into the formula, that in each concrescence whatever is determinable is determined, but that there is a remainder for the decision of the subject-superject of that concrescence. The subject-superject is the universe in that synthesis, and beyond it there is nonentity. This final decision is the reaction of the unity of the whole to its own internal determination. . . But the decision of the whole arises out of the determination of the parts, so as to be strictly relevant to it. ${ }^{106}$

It is thus by the Category of Subjective Harmony that aversions productive of diversities are the seeds of complexity rather than chaos. And it is by the Category of Subjective Intensity that a "subjective aim" toward complexity-the balance between regularity and diversity, reproduction and reversion-is, in part, generated by an 
anticipatory feeling of the relevant future. It might seem at first glance that quantum mechanics, and indeed any aspect of physics, ought solely concern itself with the immediate present as causally related to the past; but the fact that quantum mechanics terminates with probability valuations clearly evinces the relevance of Categoreal Obligations VII and VIII. For the notion of relevant future is a defining element in the concept of mathematical probability. Unlike a classical measurement, which is solely a qualification of the immediate present (which, of course, may be used to predict the relevant future, but does not in itself necessarily entail the relevant future), a quantum mechanical measurement yields probabilities, which necessarily pertain to both the immediate present and the relevant future.

As discussed earlier, the concept of a probability-valuated outcome state in quantum mechanics necessarily presupposes both facts antecedent to the measurement, as well as facts subsequent to and consequent of the measurement. That probabilities are potentia qualified as mutually exclusive and exhaustive is related to the Category of Subjective Unity; that probabilities are potentia qualified as valuations-tendencies toward reproduction or reversion-is related to the Category of Subjective Harmony and Subjective Intensity.

It follows that balanced complexity is the outcome of this final category of subjective aim. Here "complexity" means the realization of contrasts, of contrasts of contrasts, and so on; and "balance" means the absence of attenuations due to the elimination of contrasts which some elements in the pattern would introduce and other elements inhibit. . . ${ }^{107}$

It requires a complex constitution to state diversities as consistent contrasts. . . . The contrasts produced by reversion are contrasts required for the fulfillment of the aesthetic ideal. Unless there is complexity, ideal diversities lead to physical impossibilities, and thence to impoverishment. ...108

Thus there is urge towards the realization of the maximum number of eternal objects [pure potentia] subject to the restraint that they must be under conditions of contrast. ${ }^{109}$

The study of complexity in nature has been the topic of a great deal of research and debate over the past several years, and it is very 
likely that quantum mechanics describes such complexity at the most fundamental physical level. The concept of "balanced complexity" suggested by Whitehead, and its function and exemplification in his metaphysical scheme, has a direct analog in the concept of "effective complexity" - also a balance of regularity and diversity. The function and exemplification of effective complexity in quantum mechanics will likely prove to be an important avenue of inquiry in the coming years, and the decoherence-based interpretations of quantum mechanics are clearly the interpretations best suited to such an investigation. For the decoherence effect is predicated upon the very notions of contrast elucidated in Categories VII and VIIIdiverse multiplicities of facts, contrasted with regulated potential integrations of these facts-notions that clearly pertain to "balanced complexity" in nature. One might, for example, correlate the "urge towards the realization of the maximum number of eternal objects subject to the restraint that they must be under conditions of contrast" with an urge toward expressing $|\Psi\rangle$ in terms of the maximum number of potential eigenvectors, subject to the restraint of necessary contrast, such that they be mutually orthogonal and thus representative of mutually exclusive, exhaustive, potential outcome states.

\section{NOTES}

1. Alfred North Whitehead, Process and Reality: An Essay in Cosmology, Corrected Edition, ed. D. Griffin and D. Sherburne (New York: Free Press, 1978), 238-239.

2. Ibid., 7.

3. Ibid., 78.

4. Werner Heisenberg, Physics and Philosophy (New York: Harper Torchbooks, 1958), 129.

5. Alfred North Whitehead, Science and the Modern World (New York: Free Press, 1967), 47.

6. Whitehead, Process and Reality, 309.

7. Ibid., 79 .

8. Ibid., 24.

9. Ibid., 65 .

10. Ibid., 237. 
11. Ibid., 116.

12. Ibid., 239.

13. Ibid., 78-79.

14. Alfred North Whitehead, Modes of Thought (New York: Macmillan, 1938), 120.

15. Whitehead, Process and Reality, 228.

16. Ibid., 78-79.

17. This could also apply, though less obviously, to the "old" quantum theories of Einstein and Planck.

18. Whitehead, Process and Reality, 309.

19. Ibid., 65.

20. Heisenberg, Physics and Philosophy, 55-56.

21. Whitehead, Process and Reality, 78.

22. Ibid., 309.

23. Ibid., 116.

24. Ibid., 117.

25. Abner Shimony, "Quantum Physics and the Philosophy of Whitehead," Boston Studies in the Philosophy of Science, ed. R. Cohen and M. Wartofsky (New York: Humanities Press, 1965), 2:308.

26. Ibid., 322.

27. Henry Folse, "The Copenhagen Interpretation of Quantum Theory and Whitehead's Philosophy of Organism," Tulane Studies in Philosophy 23 (1974): 33.

28. Ibid., 47.

29. Whitehead, Process and Reality, 116.

30. That is, with the explicit inclusion of von Neumann's concept of nonunitary state reduction (his "Process 1") and the related mechanism of decoherence, which requires nothing more than the usual Schrödinger equation of the Copenhagen formalism.

31. That is, ontologically or "truly" closed; one can easily create "epistemologically" closed systems, as is often done in the laboratory, where a given experimental arrangement can-without the need of any complicated physical procedure- be effectively "closed" from distant and effectively unrelated environmental facts. The purpose of the ontological interpretation of quantum mechanics is to disclose the mechanism by which such epistemologically closed systems are created, and why such excluded factual relations are necessary. The decoherence effect is one such mechanism being investigated by physicists.

32. John von Neumann, Mathematical Foundations of Quantum Mechanics (Princeton, N.J.: Princeton University Press, 1955). 
33. Whitehead, Process and Reality, 285.

34. Ibid., 40.

35. Ibid., 149 .

36. Ibid., 149.

37. Ibid., 44.

38. Ibid., 43.

39. Ibid., 164.

40. Ibid., 285-286.

4l. Ibid., 24 .

42. Ibid., 65 .

43. Ibid., 84 .

44. Ibid., 84 .

45. Ibid., 45.

46. Ibid., 29.

47. Ibid., 150 .

48. Ibid., 228 .

49. Ibid., 286.

50. Ibid., 25-26.

51. Ibid., 212.

52. Ibid., 283.

53. Ibid., 212.

54. Ibid., 164.

55. Ibid., 22.

56. Ibid., 50 .

57. Ibid., 237-238.

58. Roland Omnès, The Interpretation of Quantum Mechanics (Princeton, N.J.: Princeton University Press, 1994), 344.

59. Charles Hartshorne, "Bell's Theorem and Stapp's Revised View of Space-Time," Process Studies 7, no. 3 (1977): 188.

60. Whitehead, Process and Reality, 152.

61. Ibid., 212.

62. Ibid., 65.

63. The term "mental pole" is not intended to suggest that consciousness or any sort of intellectual function be ascribed to all facts; nor should the terms "physical pole" and "mental pole" be correlated with the dualism of Descartes, especially given that the Whiteheadian philosophy is a fundamental repudiation of this dualism.

64. Whitehead, Process and Reality, 65.

65. Ibid., 23.

66. Ibid., 26.

67. Ibid., 22. 
68. Ibid., 44.

69. Ibid., 23.

70. Ibid., 249.

71. Ibid., 26.

72. Ibid., 253-254.

73. Ibid., 240.

74. Ibid., 239-240.

75. Ibid., 45.

76. Hartshorne, "Bell's Theorem," 188.

77. Whitehead, Process and Reality, 248.

78. Ibid., 101.

79. Ibid., 101.

80. The mutual exclusivity of "real" potentia (i.e., probable outcome states) is, again, a requirement of both the Whiteheadian scheme and quantum mechanics. Whitehead writes: "The definiteness of the actual arises from the exclusiveness of eternal objects in their function as determinants. If the actual entity be this, then by the nature of the case it is not that or that. The fact of incompatible alternatives is the ultimate fact in virtue of which there is definite character."

81. Whitehead, Process and Reality, 235.

82. Ibid., 26.

83. Ibid., 238.

84. Ibid., 225.

85. Ibid., 225.

86. Ibid., 227.

87. Ibid., 101.

88. Ibid., 254.

89. Ibid., 226-227.

90. Ibid., 254.

91. Ibid., 101.

92. Ibid., 34.

93. Ibid., 251.

94. Ibid., 27.

95. Ibid., 253.

96. Ibid., 254.

97. Ibid., 254.

98. Ibid., 251.

99. Ibid., 248-249.

100. Ibid., 276-277.

101. Ibid., 245.

102. Ibid., 277.

103. Ibid., 27. 
104. Ibid., 255.

105. Ibid., 27.

106. Ibid., 27-28.

107. Ibid., 278.

108. Ibid., 255 .

109. Ibid., 278. 